\title{
Performance Evaluation of Channel Access Methods for Dedicated IoT Networks
}

\author{
Abderrahman Ben Khalifa, Razvan Stanica \\ Univ Lyon, INSA Lyon, Inria, CITI, F-69621 Villeurbanne, France \\ Email: abderrahman.ben-khalifa@insa-lyon.fr
}

\begin{abstract}
Networking technologies dedicated for the Internet of Things are different from the classical mobile networks in terms of architecture and applications. This new type of network is facing several challenges to satisfy specific user requirements. Sharing the communication medium between (hundreds of) thousands of connected nodes and one base station is one of these main requirements, hence the necessity to imagine new solutions, or to adapt existing ones, for medium access control. In this paper, we start by comparing two classical medium access control protocols, CSMA/CA and Aloha, in the context of Internet of Things dedicated networks. We continue by evaluating a specific adaptation of Aloha, already used in low-power wide area networks, where no acknowledgement messages are transmitted in the network. Finally, we apply the same concept to CSMA/CA, showing that this can bring a number of benefits. The results we obtain after a thorough simulation study show that the choice of the best protocol depends on many parameters (number of connected objects, traffic arrival rate, allowed retransmission number), as well as on the metric of interest (e.g. packet reception probability or energy consumption).
\end{abstract}

\section{INTRODUCTION}

After almost two decades of research on multi-hop wireless sensor networks, we are witnessing today a paradigm shift, where small, energy constrained things are connected to the rest of the world through one-hop, cellular dedicated networks, also described as low-power wide area networks (LPWAN) [1]. Multiple technologies are competing in this dedicated Internet of Things (IoT) market, either proposed by new players (e.g. Sigfox [2], LoRa [3]), or backed up by well established standardization bodies, (e.g. NB-IoT from the $3 \mathrm{GPP}$ consortium [4], or IEEE 802.11ah from the WiFi Alliance [5]). Practically, dedicated IoT networks are characterized by a cellular architecture, with a central base station or gateway, collecting data from a number of objects in its coverage.

Another common characteristic of all these technologies is that they follow one of two classical channel access schemes: Aloha or Carrier Sense Multiple Access with Collision Avoidance (CSMA/CA). The only modification brought to these classical approaches is that, in some technologies, Aloha is modified in order to not transmit acknowledgment (ACK) messages, generally because a downlink is not always available in these networks.

Basically, three main MAC layer solutions are currently competing in the IoT world. First of all, the technologies coming from the 3GPP world, whether LTE-M or NB-IoT [6], are using classical Aloha solutions. More precisely, these technologies use a slotted-Aloha approach [7], where nodes can only access the medium on specific time-frequency blocks of the LTE random access channel.

A second class of IoT technologies, known as LPWAN, is also based on an Aloha MAC protocol. Indeed, Aloha is a very simple strategy, where the MAC layer directly transmits any message produced by the application layer. Easy to implement and without any strong synchronization requirements, Aloha was the obvious choice for new technologies, such as Sigfox [2] and LoRa [3]. However, these technologies also needed to cope with the well known poor scalability of Aloha; this was done by over-provisioning, the temporal Aloha space being expanded in the frequency domain (e.g. in Sigfox) or in the code domain (e.g. in LoRa). Moreover, these technologies are generally asymmetrical, meaning that the downlink is (very) limited. This has a direct consequence on the MAC layer, as the gateway does not acknowledge the reception of data messages from the nodes.

Finally, the most successful wireless technology nowadays, $\mathrm{WiFi}$, is also making a place in the IoT world, through a new amendment known as WiFi HaLow, and standardized as IEEE 802.11ah [5]. As all the technologies from the IEEE 802.11 family, WiFi HaLow is based on a CSMA/CA MAC layer. The main difference with respect to the classical Distributed Contention Function (DCF) used in IEEE 802.11 is that the existing stations are divided into groups, and the contention only happens between stations from the same group. The gateway defines the different time intervals for each contending group.

Our objective in this paper is not to study the specificities of each of these technologies. Instead, we want to focus on the basic properties of Aloha and CSMA/CA access strategies, in order to understand whether one of these solutions is more appropriate than the other in the IoT context.

As a matter of fact, Aloha and CSMA/CA have been well studied and compared in the literature, starting from the ' $70 \mathrm{~s}$ [8] and until the modern days [9]. However, the performance of the two schemes is generally evaluated in terms of throughput, while considering stations with saturated traffic. This is normal in wireless local wireless networks, where the objective is to have a reliable and rapid communication, generally in terms of file transfer. However, IoT scenarios are very different, characterized by dense networks with sparse, unsaturated traffic. The classic use-case in these networks is data collection, where measures of some physical phenomenon (e.g. air pollution [10] or vehicular traffic [11]) are uploaded to a central server. In this case, the throughput is no longer an essential metric, as the 
performance of the IoT applications is much better described by metrics such as the packet reception ratio.

In this paper, we focus on these IoT metrics, through an extensive simulation study, detailed in Sec. III. We compare classic Aloha and CSMA/CA in Sec. IV, showing that each of them is adapted to certain IoT scenarios and metrics. We evaluate the LPWAN flavor of Aloha, which does not use ACKs, in Sec. V. Interestingly, our results show that removing ACKs does not only conserve energy, but it can even improve the packet reception probability. Finally, for the first time in the literature, we apply the same concept of removing ACKs to CSMA/CA in Sec. VI, showing interesting performance gains.

\section{RELATED WORK}

Aloha and CSMA/CA are among the first MAC protocols to be proposed in the history of wireless networks. As a consequence, they have been thoroughly studied and compared in the last four decades [8]. However, despite considering the impact of numerous parameters, such as the buffer size [12] or the number of retransmissions [13], most of these studies focused on scenarios with saturated stations, i.e. users who always have packets to transmit to one another. This is quite different, both in terms of system functioning and metrics, from the dedicated IoT networks we are interested in.

While new theoretical tools, e.g. based on stochastic geometry [9], were developed recently, the studied scenarios remained generally the same. Even in the few studies where Aloha and CSMA/CA were compared in scenarios with unsaturated traffic [14], the system throughput was still used as the main metric. On the other side, in the cases where an evolution can be noticed in terms of the studied metrics (e.g. the distribution of the channel access delay [15]), saturated traffic is considered.

In related fields, where new wireless network architectures are imagined, e.g. in mobile ad hoc networks [16] or in vehicular networks [17], Aloha and CSMA/CA are always among the first MAC solutions to be tested. However, these studies remain focused on throughput in saturated traffic conditions.

Regarding dedicated IoT networks, two recent studies [18], [19] evaluate the performance of Aloha-based techniques. Li et al. [18] take a stochastic geometry approach, focusing on throughput and on the outage probability of a station, defined as the probability that a station observes a signal to noise and interference ratio (SINR) above a certain threshold. Song et al. [19] argue that packet delivery ratio is a more suitable metric in IoT use-cases, and they study the performance of slottedAloha with respect to this metric. However, the only parameter considered in [19] is the packet arrival rate.

Most of these studies focus on analytical models, which are essential tools in the understanding of a network protocol. However, this type of mathematical modeling usually requires some important simplifications: e.g. the use of slotted-Aloha instead of Aloha [19], independence of retransmissions [16], or simplified CSMA/CA back-off [9]. Instead, we propose a thorough simulation study, removing most of these artificial assumptions, in order to evaluate different MAC solutions in dedicated IoT networks.

\section{Simulation Methodology}

We use the Network Simulator 3 (ns3) to study a dedicated IoT network with $N$ sender nodes and one sink node. The network topology is a circle with radius $r$ and the base station sink node is its center, while the sender nodes are uniformly distributed inside this area and they all share the same channel.

Each sender node produces one packet of data each time period $T$, while the sink node only transmits ACK frames (in case the MAC protocol uses them). Different IoT technologies achieve very different data rates at the physical layer, from 100 $\mathrm{b} / \mathrm{s}$ in Sigfox to several Mb/s in WiFi HaLow. In order to have a fair, but technology agnostic comparison, we are using as a parameter the transmission opportunity, $T_{o p}=S / T$, where $S$ is the airtime of a MAC layer frame. As an example, a $T_{o p}$ value of $165 \cdot 10^{-6}$ corresponds to a packet arrival every second in WiFi HaLow and every 20 minutes in SigFox.

Unlike previous studies, which mostly focus on the throughput as an evaluation metric, we use metrics more relevant for the IoT context: the packet success probability and the time each node spends in an ON state (receiving, transmitting or listening to the channel). We consider that this second metric is a good generic proxy for the energy consumption of a node.

\begin{tabular}{|c||c|}
\hline \multicolumn{1}{|c||}{ Parameter } & Value \\
\hline Acknowledgement Timeout & $75 \mathrm{~ms}$ \\
\hline Maximum Number of Retransmission & 7 \\
\hline RTS/CTS message exchange & Disabled \\
\hline Frequency & $5.180 \mathrm{GHz}$ \\
\hline Transmission Power & $16 \mathrm{dBm}$ \\
\hline Clear Channel Assessment Threshold & $-99 \mathrm{dBm}$ \\
\hline Propagation Loss Model & Log Distance \\
\hline Propagation Delay Model & Constant Speed Mode \\
\hline Transmission Data Rate & $6 \mathrm{Mbps}$ \\
\hline Transmission Opportunity & $165 \cdot 10^{-6}$ \\
\hline
\end{tabular}

TABLE I

DEFAULT SIMULATION PARAMETERS.

For the CSMA/CA protocol, we used the ns3 AdhocWifiMac as a MAC layer model, using the parameters indicated in Tab. I. Since ns3 does not directly provide an Aloha implementation ${ }^{1}$, we adapted the same AdhocWifiMac model used in the CSMA/CA case. This allowed us to have the same basis implementation for the two solutions, for a fair evaluation. The first adaptation required in order to model Aloha was to deactivate the clear channel assessment (CCA) mechanism, which implements the carrier sensing operation. Since Aloha does not use carrier sensing, we set the CCA threshold to an infinite value, so that the node always considers the channel as free and the transmissions are never blocked. Another adaption consists in deactivating the random backoff used in CSMA/CA before a new transmission; we do

\footnotetext{
${ }^{1} \mathrm{~ns} 3$ provides an implementation of the Aloha protocol in the Uan package, used to simulate underwater acoustic communications. This implementation is not compatible with radio communication models in the simulator.
} 
this by setting the maximum contention window to 0 in AdhocWifiMac.

We run simulations while varying the number of sender nodes. Every simulation lasts 30 seconds and it is repeated 10 times, with a different seed value each time. All the results presented in the remainder of the paper are shown with a confidence interval of $95 \%$.

\section{AlOHA AND CSMA/CA COMPARISON}

We begin our study by comparing the classical Aloha and CSMA/CA solutions in a dedicated IoT context. We evaluate the packet success probability and the time spent by the nodes in an active $\mathrm{ON}$ state, while also looking at the impact of the number of authorized retransmissions.

\section{A. Packet Success Probability}

The essential performance metric in IoT networks is the success probability of a message. The small size of the messages transmitted by the nodes allows their encapsulation into single packets, which are possibly transmitted multiple times as MAC layer frames. Since we are interested in the overall performance of the MAC layer, in Fig. 1 we present the average packet success probability of the two MAC protocols studied in this section, calculated for different number of contending nodes and packet arrival periods (given as a varying number of transmission opportunities).

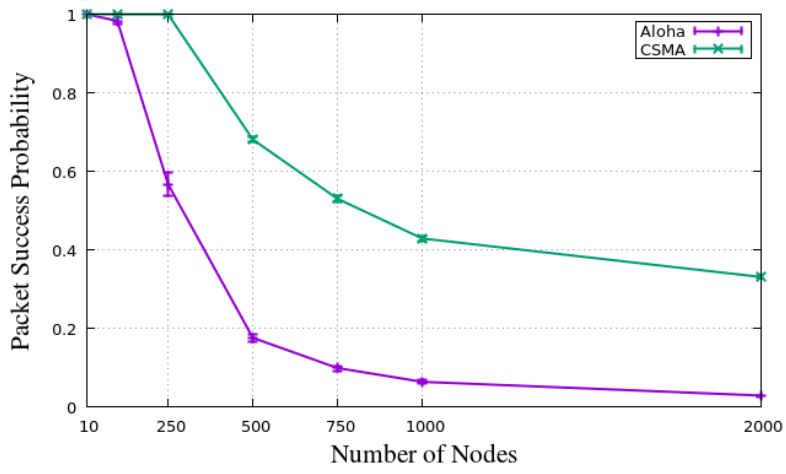

(a) $T_{o p}=165 \cdot 10^{-5}$

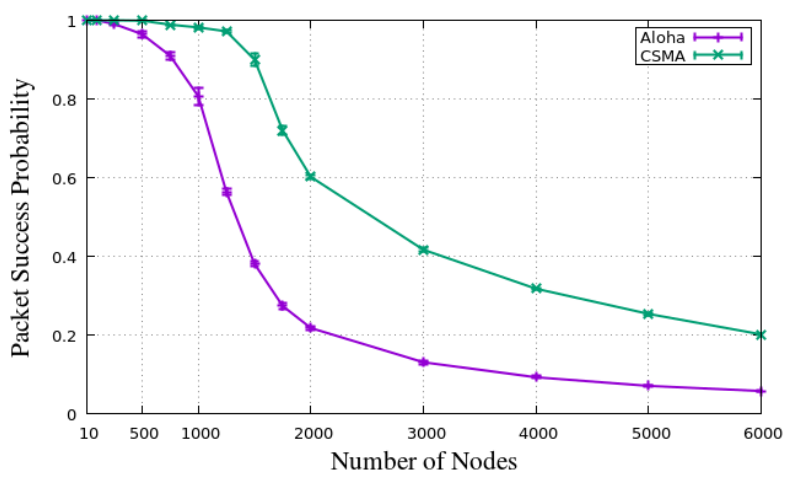

(b) $T_{o p}=165 \cdot 10^{-6}$

Fig. 1. Packet success probability for Aloha and CSMA/CA in an IoT context.

In Fig. 1a, showing the packet success probability obtained for a transmission opportunity $T_{o p}=165 \cdot 10^{-5}$ and for different number of nodes, we can distinguish two different regions. In the first region, for small networks up to 100 nodes, the CSMA/CA and Aloha success probabilities are both equal or near to 1 . In the second region, for medium size and dense networks, the performance of the two protocols starts decreasing, with CSMA/CA getting the best results. This is expected, as CSMA/CA is using a CCA mechanisms to reduce the number of collisions and a back-off technique to avoid their repetition.

To complement these results, we also show the packet success probability for a transmission opportunity value ten times smaller in Fig. 1b. This corresponds to scenarios with a more reduced packet arrival rate. A similar trend can be observed, but the capacity of the network, in terms of number of nodes, increases.

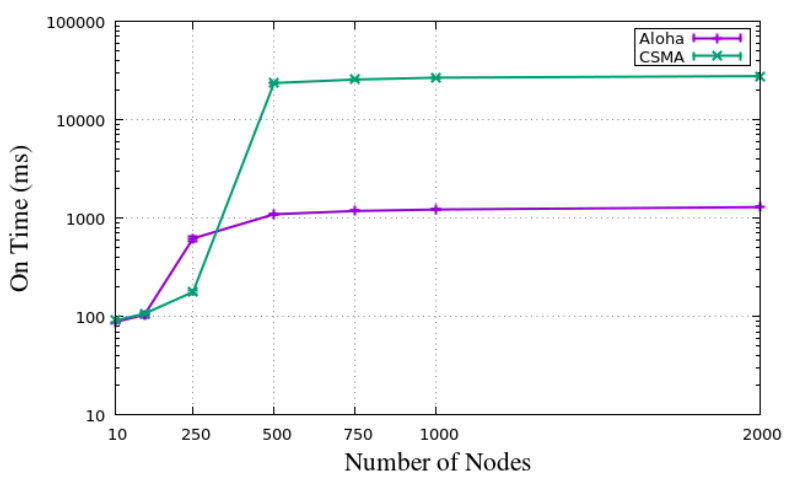

(a) $T_{o p}=165 \cdot 10^{-5}$

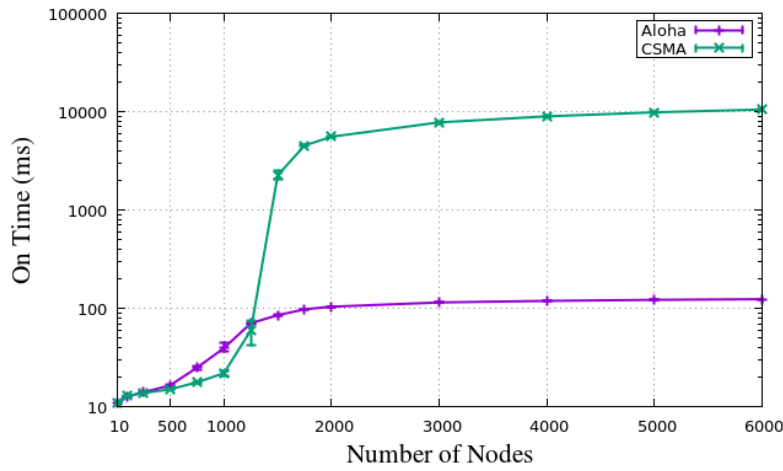

(b) $T_{o p}=165 \cdot 10^{-6}$

Fig. 2. Average ON time for Aloha and CSMA/CA in an IoT context (please note the $\log$-scale of the $y$-axis).

\section{B. Node ON Time}

The energy consumption is an important metric for most IoT devices, constrained in terms of size, hence battery. In this work, we do not directly compute the node energy consumption, as this would limit us to the numeric values of a particular technology. Instead, we calculate a correlated metric: the duration each node spends in an ON state, i.e. the time the node is using its radio module, either for transmission, reception or listening the channel. In the case of Aloha, we consider that the node is continuously listening the channel 
while waiting for an ACK message. In the case of CSMA/CA, we consider that the node is ON to sense the channel during the back-off slots and after the transmission, listening to the channel waiting for an ACK message.

Fig. 2 shows the average ON time for the two protocols, for two values of the transmission opportunity parameter and for different number of nodes. We observe that, for a low density network, both protocols have almost the same energy consumption, because we are not facing any collision or CSMA/CA back-off yet. Interestingly, as the number of nodes increases, CSMA/CA becomes more energy-friendly than Aloha for a while, since the latter solution results in more collisions and therefore requires more retransmissions. However, after a certain threshold, Aloha demonstrates a much better performance, consuming 10 times less energy than CSMA/CA. This trend is observed for both values of $T_{o p}$, in Fig. 2a and in Fig. $2 b$.

\section{Retransmission Number Impact}

Both CSMA/CA and Aloha use backoff-based retransmissions in case of a missing ACK. In CSMA/CA, the retransmissions follow a binary exponential back-off, which increases for each missing ACK. When the maximum number of retransmissions $R$ is reached, the message is dropped and the packet is considered as lost. We simulated Aloha and CSMA/CA with a maximum number of retransmission of 3, 5 and 7, while keeping the other default MAC layer parameters from Tab. I.

Fig. 3a shows the packet success probability with a variable maximum number of retransmission, showing the significant impact of this parameter. For Aloha, we notice that the lower the number of retransmissions, the better the performance of the protocol. This is because a higher number of authorized retransmissions increases the collision probability. Aloha is also obtaining the best energy consumption results with the lowest number of retransmissions, as shown in Fig. $3 b$.

For CSMA/CA, the results are more mitigated: the packet success probability first decreases with $R$, just as in Aloha, but it then increases again (CSMA/CA with $R=7$ gives the best performance in Fig. 3a). This is the consequence of two opposite behaviors: the increased overall collision probability with more retransmissions and the binary exponential back-off which reduces the collision probability for higher retransmission indices.

We can see as well that CSMA/CA is obtaining better results than Aloha almost all the time in terms of reliability, except with $R=5$ for a network of 250 nodes. This comes with an energy price, as CSMA/CA consumes more energy in networks of more than 250 nodes and this energy consumption increases with $R$ (please note the log-scale on the y-axis in Fig. $3 b)$. However, we still observe that CSMA/CA energetically outperforms Aloha for networks of 10 to 250 nodes.

From all these results, we can conclude that the best choice between Aloha and CSMA/CA in an IoT context depends on the metric we want to satisfy: Aloha gives better results

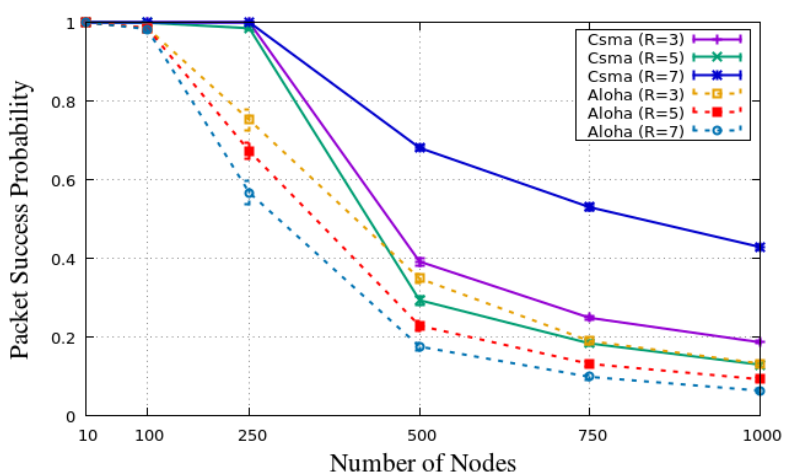

(a) Packet Success Probability

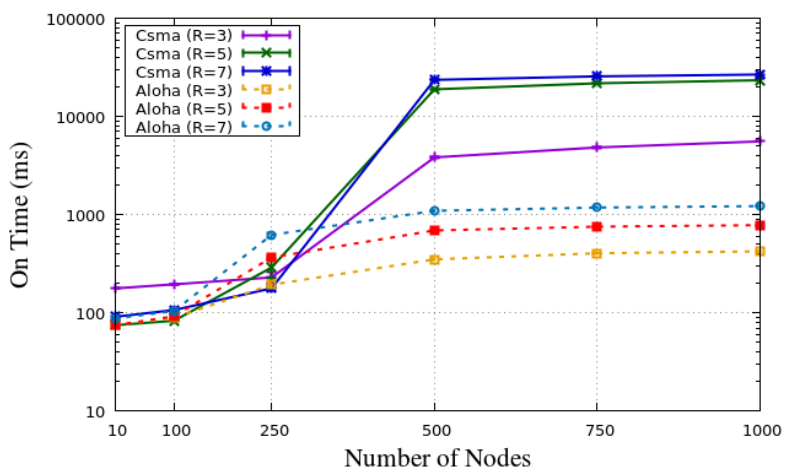

(b) Average ON Time (ms) (y-axis on log-scale)

Fig. 3. Impact of the maximum number of retransmissions in Aloha and CSMA/CA.

energy-wise, while CSMA increases the packet success probability. The environment, e.g. the number of nodes and the packet arrival rate, also plays a major impact on the protocol choice, and so are the different parameters of the two protocols (maximum number of retransmissions, back-off mechanism).

\section{Aloha For Dedicated IoT Networks}

As explained above, Aloha has been adapted to LPWAN technologies by removing the ACK messages. This mainly comes from a limitation of LPWAN solutions, where a downlink is not always available [1]. Instead, for reliability reasons, $K$ copies of each message are transmitted by the nodes.

In this context, we study the impact of the absence of ACK messages, as well as that of the number of transmitted copies $K$, on the network performance. For this, we simulate a modified version of Aloha, under the same conditions as the previous simulations. This modified Aloha version, which we denote as Aloha No Ack, sends each packet $K$ times at the MAC layer, without waiting for any ACK message. For example, Sigfox currently uses a static value of $K=3$ in their system. For the case of $K=1$, the node sends the packet only once and goes back to sleep. For the case of $K>1$, the node wakes up and sends the packet once, and then sleeps a random number of slots before waking up again to send the next copy of the packet.

Fig. 4 shows the results of transmission reliability and energy consumption obtained when comparing Aloha No Ack 


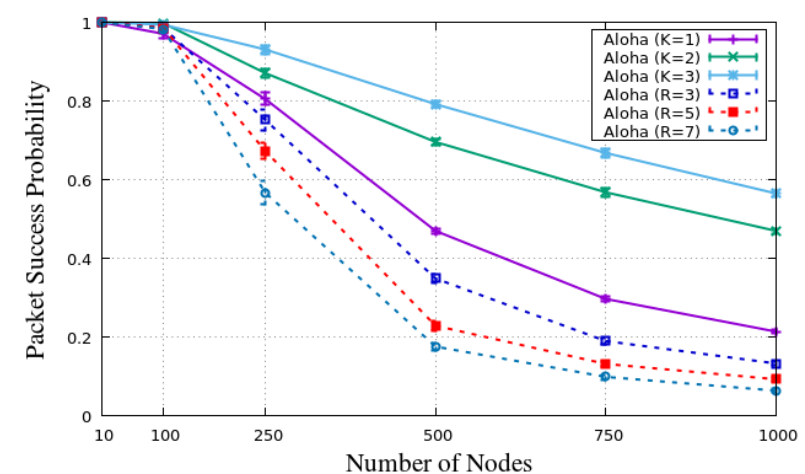

(a) Packet Success Probability

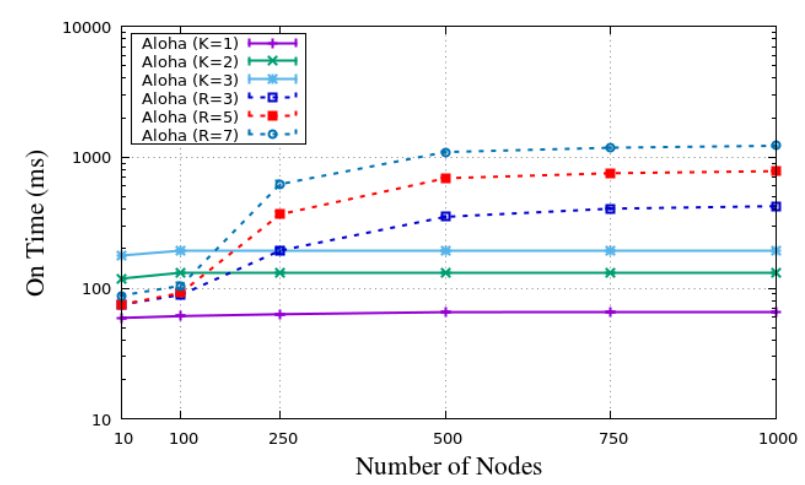

(b) Average ON Time (ms) (y-axis on log-scale)

Fig. 4. Comparison of classical Aloha and Aloha No Ack.

and classical Aloha. We remark that removing the ACK messages is not only a necessity to cope with a missing downlink, but it actually improves the packet success probability. Indeed, Aloha No Ack with $K=3$ is outperforming all the other Aloha and Aloha No Ack flavors in terms of reliability.

On the energy consumption side, Aloha No Ack has a constant ON time, which only depends on the number of transmitted copies $K$ and it can be computed as $K \cdot S$. Aloha No Ack with $K=3$ consumes more energy than classical Aloha for a network with less than 250 nodes, but it outperforms the ACK-based approach for denser networks.

We note that ACK messages are considered as a prominent mechanism for reliability purposes at the MAC layer. In any throughput-focused solution, ACKs represent an essential feed-back to the transmitter. However, the results we obtained in this section demonstrate that removing ACKs actually brings benefits in IoT networks, when focusing on metrics such as packet success probability and energy consumption.

\section{CSMA/CA FOR DEDiCATEd IOT NeTWORKS}

Similarly to how Aloha has been adapted for LPWANs, we propose removing ACKs from CSMA/CA as well. This solution, similar to what is used to transmit broadcast traffic in WiFi networks [20], has not been priorly tested in IoT scenarios. In this solution, denoted as CSMA No Ack, we use a similar parameter for the number of transmitted copies, $K$. Practically, a node wakes up to transmit a copy of the message and follows the CCA procedure. If the medium is detected as busy, the node transmits at the end of the back-off procedure. In order to transmit the next copy of the message, the procedure is repeated. However, in order to avoid collisions, a back-off procedure is needed between the transmission of two copies. Since no ACK messages are used, the contention window used by the back-off mechanism, denoted as $C W$, can not be dynamically adapted.

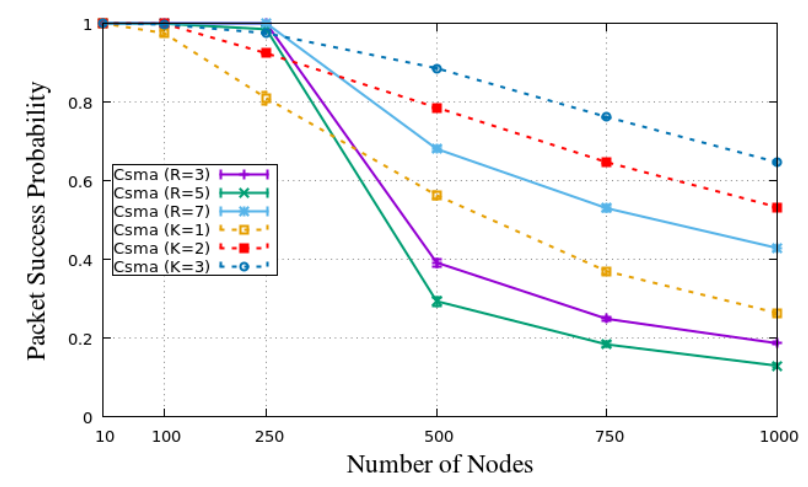

(a) Packet Success Probability

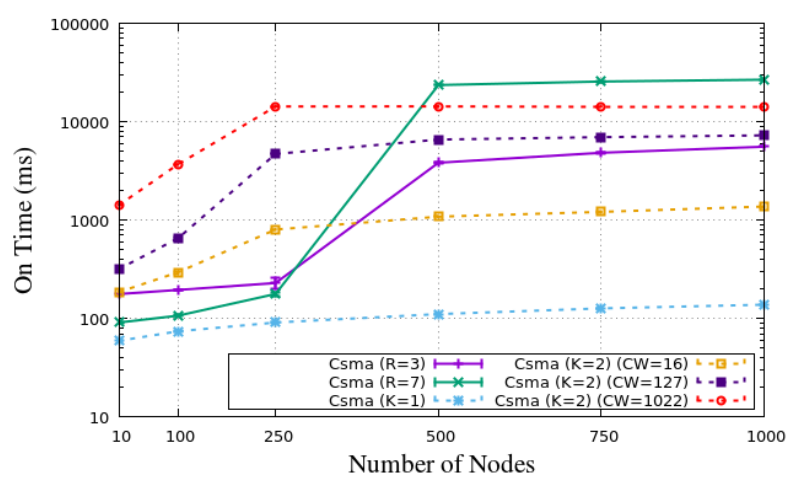

(b) Average ON Time (ms) (y-axis on log-scale)

Fig. 5. Comparison of classical CSMA and CSMA No Ack.

In Fig. 5, we compare the performance of the classical CSMA/CA and the modified CSMA/CA version, where no ACK messages are used. For the packet success probability, shown in Fig. 5a, classical CSMA/CA obtains better performance for a network with less than 250 nodes. When the network density increases, we observe a phenomenon similar to the Aloha case: removing the ACK messages actually reduces congestion on the channel and allows CSMA No Ack to obtain up to $20 \%$ better performance than classical CSMA/CA. On the energy side (Fig. 5b), CSMA No Ack has an almost constant behavior: the slight increase one can notice for CSMA No Ack with $K=1$ is a consequence of the longer time required by the back-off procedure under high network density. On the other side, the classical approach shows a significant energy consumption increase in dense networks, where more retransmissions are needed.

An important parameter for CSMA No Ack is the size of the contention window, $C W$, used between the transmission of two message copies. Fig. 5b shows the impact of this 
parameter on the active $\mathrm{ON}$ time of a node, for $K=2$. Basically, the higher $C W$, the higher the node ON time and the higher its energy consumption.

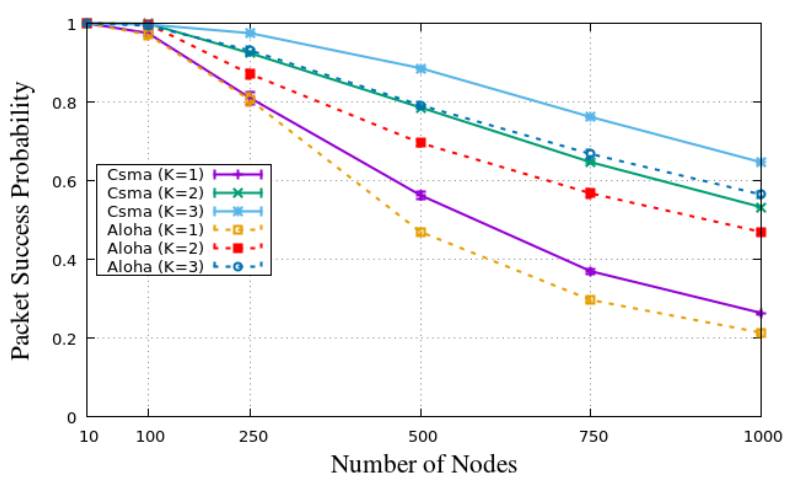

(a) Packet Success Probability

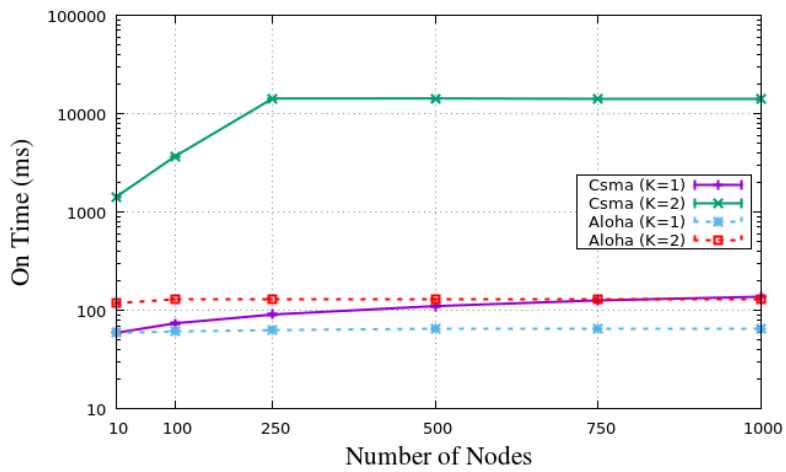

(b) Average ON Time (ms) (y-axis on log-scale)

Fig. 6. Comparison of Aloha No Ack and CSMA No Ack.

In Fig. 6, we compare the two modified versions, Aloha No Ack and CSMA No Ack. Fig. 6a shows the packet success probability for the two protocols, with a $K$ varying from 1 to 3. The simple fact of using a CCA mechanism before a transmission improves the packet success probability by close to $10 \%$ in high dense networks. However, this comes with a price on the energy side, as shown in Fig. 6b. For $K=$ 1, CSMA No Ack only spends the extra-energy of a CCA mechanism. However, when $K$ increases, the cost of the backoff mechanism is added and the consumption of CSMA/CA can reach up to $100 \mathrm{x}$ the consumption of Aloha.

\section{CONCLUSIONS}

In this paper, we discuss MAC layer solutions for dedicated IoT networks and evaluate them through ns3 simulation. We show that the best MAC layer approach depends on parameters such as the number of competing nodes and the packet arrival rate. In addition, we remark that using ACK frames can have a negative impact, always reducing the network performance in terms of reliability and energy consumption.

Following this study, we believe that the MAC strategy for dedicated IoT network needs to adapt to the network state and to the target metric. Moreover, depending on the local traffic density, different MAC solutions can be used in different parts of the cell, orchestrated by a smart gateway. We will explore such approaches in our future work.

\section{ACKNOWLEDGMENT}

This work has been partly supported by the INSA Lyon SPIE ICS chair on the Internet of Things.

\section{REFERENCES}

[1] U. Raza, P. Kulkarni, M. Sooriyabandara, "Low Power Wide Area Networks: An Overview", IEEE Communications Surveys \& Tutorials, vol. 19, no. 2, pp. 855-873, Apr. 2017.

[2] Y. Mo, C. Goursaud, J.M. Gorce, "Theoretical Analysis of UNB-based IoT Networks with Path Loss and Random Spectrum Access", Proc. IEEE PIMRC 2016, Valencia, Spain, Sep. 2016.

[3] O. Iova, A.L. Murphy, G.P. Picco, L. Ghiro, D. Molteni, F. Ossi, F. Cagnacci, "LoRa from the City to the Mountains: Exploration of Hardware and Environmental Factors", Proc. MadCom 2017, Uppsala, Sweden, Feb. 2017.

[4] E. Wang, X. Lin, A. Adhikary, A. Grovlen, Y. Sui, Y. Blankenship, J. Bergman, H.S. Razaghi, "A Primer on 3GPP Narrowband Internet of Things", IEEE Communications Magazine, vol. 55, no. 3, pp. 117-123, Mar. 2017.

[5] T. Adame, A. Bel, B. Bellalta, J. Barcelo, M. Oliver, "IEEE 802.11ah: The WiFi Approach for M2M Communications", IEEE Wireless Communications, vol. 21, no. 6, pp. 144-152, Dec. 2014.

[6] M. Lauridsen, I.Z. Kovacs, P. Mogensen, M. Sorensen, S. Holst, "Coverage and Capacity Analysis of LTE-M and NB-IoT in a Rural Area", Proc. IEEE VTC Fall, Montreal, QC, Canada, Sep. 2016.

[7] S. Cherkaoui, I. Keskes, H. Rivano, R. Stanica, "LTE-A Random Access Channel Capacity Evaluation for M2M Communications", Proc. IFIP WD, Toulouse, France, Mar. 2016.

[8] L Kleinrock, F.A. Tobagi, "Packet switching in radio channels: Part ICarrier sense multiple-access modes and their throughput-delay characteristics", IEEE transactions on Communications, vol. 23, no 12, p. 14001416, Dec. 1975.

[9] B. Blaszczyszyn, P. Muhlethaler, S. Banaouas, "A Comparison of ALOHA and CSMA in Wireless Ad Hoc Networks under Different Channel Conditions", Inria Research Report 00530093, Oct. 2010.

[10] A. Boubrima, F. Matigot, W. Bechkit, H. Rivano, A. Ruas, "Optimal Deployment of Wireless Sensor Networks for Air Pollution Monitoring”, Proc. ICCCN, Las Vegas, NV, USA, Aug. 2015.

[11] R. Domga Komguem, R. Stanica, M. Tchuente, F. Valois, "WARIM: Wireless Sensor Network Architecture for a Reliable Intersection Monitoring", Proc. IEEE ITSC, Qingdao, PRC, Oct. 2014.

[12] T. Saadawi, A. Ephremides, "Analysis, Stability and Optimisation of Slotted Aloha with a Finite Number of Buffered Users", IEEE Transactions on Automatic Control, vol. 26, no. 3, pp. 680-689, Jun. 1981.

[13] D. Raychaudhuri, K. Joseph, "Performance Evaluation of Slotted Aloha with Generalized Retransmission Backoff", IEEE Transactions on Communications, vol. 38, no. 1, pp. 117-122, Jan. 1990.

[14] L. Dai, "Toward a Coherent Theory of CSMA and Aloha", IEEE Transactions on Wireless Communications, vol. 12, no. 7, pp. 3428-3444, Jul. 2013.

[15] Y. Yang, T.-S. Yum, "Delay Distributions of Slotted ALOHA and CSMA", IEEE Transactions on Communications, vol. 51, no. 11, pp. 1846-1857, Nov. 2003.

[16] F. Baccelli, B. Blaszczyszyn, P. Muhlethaler, "Stochastic Analysis of Spatial and Opportunistic Aloha", IEEE Journal of Selected Areas in Communications, vol. 27, no. 7, pp. 1105-1119, Sep. 2009.

[17] A. Sakata, T. Yamazato, H. Okada, M. Katayama, "Throughput Comparison of CSMA and CDMA slotted ALOHA in Inter-Vehicle Communication", Proc. ITST, Sophia Antipolis, France, Jun. 2007.

[18] Z. Li, S. Zozor, J.-M. Drossier, N. Varsier, Q. Lampin, "2D TimeFrequency Interference Modelling using Stochastic Geometry for Performance Evaluation in Low-Power Wide-Area Networks", Proc. IEEE ICC, Paris, France, May 2017.

[19] Q. Song, X. Lagrange, L. Nuyami, "An Analytical Model for S-ALOHA Performance Evaluation in M2M Networks", Proc. IEEE ICC, Paris, France, May 2017.

[20] X. Ma, X. Chen, "Performance Analysis of IEEE 802.11 Broadcast Scheme in Ad Hoc Wireless LANs", IEEE Transactions on Vehicular Technology, vol. 57, no. 6, pp. 3757-3768, Nov. 2008. 